\title{
ARF3 inhibits proliferation and promotes apoptosis in gastric cancer by regulating AKT and ERK pathway
}

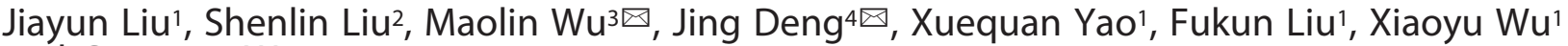 \\ and Guannan Wu'
}

1Department of Digestive Tumor Surgery, Jiangsu Province Hospital of Chinese Medicine, Affiliated Hospital of Nanjing University of Chinese Medicine, Nanjing, Jiangsu Province, 210029, China; 2 Department of Oncology, Jiangsu Province Hospital of Chinese Medicine, Affiliated Hospital of Nanjing University of Chinese Medicine, Nanjing, Jiangsu Province, 210029, China; ${ }^{3}$ Department of Oncology, Hospital of Chengdu University of Traditional Chinese Medicine, Chengdu, Sichuan Province, 610075, China; ${ }^{4}$ Department of Otolaryngology, Hospital of Chengdu University of Traditional Chinese Medicine, Chengdu, Sichuan Province, 610075, China

\begin{abstract}
ADP-ribosylation factor 3 (ARF3) has confirmed participate in diverse biological processes in many cancers. However, the expression patterns and roles of ARF3 in gastric cancer (GC) remains largely unknown. In our study, by using qRT-PCR and western blot, we found that, in In GC tissues and cells, the expression of ARF3 was significantly down-regulated. Functional experiments demonstrated that ARF3 inhibited proliferation, induced cycle arrest and enhanced apoptosis of GC cells. Moreover, by performing western blot, we found that ARF3 could regulate the protein expression of key factors of AKT and ERK pathway. Using orthotopic xenograft mouse models, it is showed that ARF3 could inhibit GC tumorigenesis in vivo. To sum up, ARF3 may suppress proliferation, induced cycle arrest and promotes apoptosis of GC by modulating AKT and ERK pathway. It might act as a potential biomarker for GC prognosis.
\end{abstract}

Keywords: ARF3; proliferation; apoptosis; Akt; ERK; gastric cancer

Received: 15 October, 2020; revised: 18 November, 2020; accepted: 19 November, 2020; available on-line: 13 April, 2021

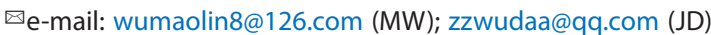
Abbreviations: ARF3, ADP-ribosylaton factor 3; GC, gastric cancer

\section{INTRODUCTION}

Gastric cancer (GC) is considered as a malignant disease with high mortality rate and limited therapeutic options (Strong 2018). Epidemiologic studies showed that GC caused more than 800000 death each year for the last decades worldwide (Ferro et al., 2014). The incidence of GC is declining in recent years mainly due to the decrease of Helicobacter pylori ( $H$. pylori) infection rate and improvements of food preservation (Lunet et al., 2005; Peleteiro et al., 2012). However, it is still an important disease which caused great burden globally (Charalampakis et al., 2018). Despite the great advanced breakthroughs that have been made in this field, the five-year survival rate remains not optimistic due to the high recurrence and distal metastasis rates (Fujiya et al., 2018; Zhang et al., 2019). Therefore, exploring new molecular mechanisms of the occurrence and development of gastric cancer is necessary.

ADP-ribosylation factor (ARFs) are classified as three classes, including ARF1, ARF2, ARF3, ARF4, ARF5, ARF6 (Li et al., 2002; Kahn et al., 2006). As a key regulator of subcellular compartments' formation, ARFs play an important role in vesicular trafficking and activating phospholipase D (Seo et al., 2020). Among them, ARF3 is one of the most important members of ARF gene family (Kondo et al., 2012). Unlike other ARFs, ARF3 contains five exons and four introns, and associates selectively with recruitment of the Golgi shell complex and activates phospholipase D and PI kinase (Smith et al., 1995; Sztul et al., 2019). Accumulating evidence suggested that ARF3 may play an essential role in cancer development (Chang et al., 2009; Zhang et al., 2019). Recent studies have shown that the expression of ARF3 is positively correlated with the clinical staging of breast cancer (Huang et al., 2019). Huang et al identified that the expression of both ARF3 mRNA and protein are highly expressed in breast cancer cells and mainly localized in cytoplasm. Mechanically, ARF3 may promote breast cancer cell proliferation by regulating cell cycle G1-S transition and associated with inactivation of the forkhead box O1 (FOXO1) transcription factor (Huang et al., 2019). In GC related study, by using microarray assays, Chang et al found that APF3 is one of the hub genes for regulating liver metastasis of gastric cancer (Chang et al., 2009). However, the mechanism of how ARF3 exert its role in GC remains not fully understood.

In this study, we aim to explore the function and mechanism in ARF3 on GC progression. The expression of ARF3 was tested in both GC human tissues and cell lines. Furthermore, by using gain- and loss- of function experiments, the role of ARF3 was clarified in GC cells. Next, the mechanism of ARF3 and its downstream factors was investigated. Finally, orthotopic xenograft mouse were used to clarify the effect of ARF3 on GC tumorigenesis in vivo. Our findings might set a novel insight into pathophysiologic mechanism of GC and may provide new therapeutic options for treating GC.

\section{MATERIALS AND METHODS}

\section{GC tissue collection and GC cell culture}

The study (human part) was approved by the ethical committee of the Jiangsu Province Hospital of Chinese Medicine. Signed informed consent was collected from each patient enrolled in this study prior to any medical related procedure. A total number of 61 pairs of gastric tumor and corresponding adjacent tissue were collected from patients with gastric cancer receiving surgical resection. All tissue specimens were labeled and kept at $-80^{\circ} \mathrm{C}$ for further use. 
For GC cell culture, human GC cell lines (HGC-27, AGS, SNU-1, MKN-45) and normal gastric mucosal cells line (GES-1) were obtained from American Type Culture Collection (ATCC). All GC cells were cultured in standard DMEM medium (Gibco, USA) which contained 10\% fetal bovine serum (FBS, Invitrogen, Carlsbad, CA, USA) and penicillin/streptomycin ((Invitrogen, USA). The culture was kept at the standard environment of $37^{\circ} \mathrm{C}$ with $5 \% \mathrm{CO}_{2}$.

\section{qRT-PCR}

Total RNA from human tissues and cells was extracted using a TRIZOL Reagent (Invitrogen, USA). Then, cDNA was synthesized using Promega cDNA kit (Madison, WI, USA). Subsequently, quantitative realtime PCR was performed on ABI 7500 Fast system using SYBR Green Mix (TaKaRa, Kusatsu, Janpan). GAP$\mathrm{DH}$ was considered as control. Data were normalized and analyzed using ${ }^{2-\Delta \Delta} \mathrm{CT}$ method. The primers were listed as following: ARF3 forward, 5'-GGAACAAGCCCCAACCGG-3', reverse, 5'-CTAAAATCAGGGGTCCCAACTG-3'; GAPDH forward, 5'-CCGGGAAACTGTGGCGTGATGG-3', reverse, 5'-AGGTGGAGGAGTGGGTGTCGCTGT'T-3'.

\section{Western blot assay}

Human tissues and transfected cells were lysed. Then, protein concentration was examined with the use of BCA Protein Assay Kit (Thermo, USA). The Western blotting analysis was performed according to standard procedures as previously described. Primary anti-ARF3 (\#ab108347, 1:1000), anti-cyclin-D1 (\#ab16663, 1:1000), anti-p21 (\#ab109520, 1:1000), anti-p27 (\#ab32034, 1:2000), antiBcl-2 antibodies (\#ab32124, 1:2000), anti-Bax (\#ab32503, 1:1000), anti-Cleaved-caspase-3 (\#ab32042, 1:1000), anti-p-AKT (\#ab38449, 1:500), anti-AKT (\#ab8805, 1:1000), anti-p-ERK1/2 (\#ab223500， 1:1000), ERK1/2 (\#ab184699, 1:1000), GAPDH (\#ab8245, 1:500), were purchased from Abcam (Cambridge, MA, USA). Secondary antibodies were anti-mouse and anti-rabbit.

\section{Immunohistochemistry assay (IHC)}

Tissue specimens were fixed in 10\% formaldehyde, embedded in paraffin. The sections $(4 \mu \mathrm{m})$ were subjected to gradient dehydration in ethanol solution. After antigen retrieval, primary anti-ARF3 antibody (Abcam, \#ab108347, 1:500) and anti-Ki-67 antibody (Abcam, \#ab15580, 1:1000) were added and co-incubated with sections at $4^{\circ} \mathrm{C}$ overnight.

\section{Cell transfection}

For generating pRNAT-U6.1/Neo-ARF3 plasmid (sh1-ARF3, sh2-ARF3), self-complementary hairpin DNA oligonucleotides were annealed and cloned into the pRNAT-U6.1/Neo plasmid vector, and NC-shRNA was used as a control. To overexpress ARF3, pcDNA3.1 plasmid targeting ARF3 (pcDNA3.1-ARF3) were purchased from Beyotime (Wuhan, China). For cell transfection, MKN-45 cells were transfected with sh-ARF3 or NC-shRNA, HGC-27 cells were transfected with pcDNA3.1-ARF3 or control plasmid with Lipofectamine 2000 (Invitrogen, Waltham, MA, USA) for 48 hours.

\section{Cell counting kit-8 (CCK-8) assay}

CCK-8 reagent was added in a 96-well plate with transfected cells (2000 cells per well). Cells were incubat- ed with reagent at $37^{\circ} \mathrm{C}$ for $4 \mathrm{~h}$. The absorbance values at $490 \mathrm{~nm}$ were detected and recorded with a microplate reader at $0 \mathrm{~d}, 1 \mathrm{~d}, 2 \mathrm{~d}, 3 \mathrm{~d}$, and $4 \mathrm{~d}$ after incubation. Data were recorded and cell viability in each group was analyzed.

\section{Edu staining}

Different group of transfected cells were suspended at a density of $1 \times 10^{5}$ cells/well. Then, $1 \times$ Apollo reaction cocktail $(100 \mu \mathrm{l} /$ well $)$ was added in culture medium for half an hour. Subsequently, DNA was stained with 10 $\mu \mathrm{g} / \mathrm{ml}$ of Hoechst 33342 stain $(100 \mu \mathrm{l} /$ well), DAPI was used for nuclei staining. The percentage of EdU positive cells were quantified and analyzed.

\section{Flow cytometer assay}

For cell cycle measurement, transfected cells were collected at logarithmic growth phase, digested with trypsin, and fixed with precooled ethanol at $4^{\circ} \mathrm{C}$ overnight. Subsequently, cells were centrifuged, washed, and re-suspended. Then, cells were stained with PI (400 $\mu$ l, Sigma) and RNAse A $\left(0.1 \mathrm{mg} / \mathrm{ml}\right.$, Sigma) for $30 \mathrm{~min}$ at $37^{\circ} \mathrm{C}$ in the dark. After that, the cell cycle was detected by flow cytometry.

For apoptotic assay, transfected cells were digested with trypsin (without EDTA) and washed and re-suspended in $100 \mu \mathrm{l}$ binding buffer. Then, $5 \mu \mathrm{l}$ Annexin V/ FITC and $5 \mu \mathrm{l}$ propidium iodide (PI) were added in the dark for $15 \mathrm{~min}$ at room temperature. Cell apoptosis was detected by flow cytometry.

\section{Tumor xenograft experiments}

HGC-27 cells $\left(2 \times 10^{6}\right)$ transfected with pcDNA3.1ARF3 plasmid or control vector were subcutaneously injected in the right flank of male $\mathrm{BALB} / \mathrm{c}$ nude mice ( $\mathrm{n}=6$ for each group, 6 8 weeks old, 19 22g). Tumor volume was calculated every 3 days up to 18 days. At the end of the18th day, mice were sacrificed, and the weight of the tumors was recorded. Animal-related study was approved by the ethical committee of the Jiangsu Province Hospital of Chinese Medicine.

\section{Statistical analysis}

All series of experiments were performed at least 3 times and results were presented as the mean \pm standard deviation. Statistical analyses were performed on SPSS version 23.0 (IBM, Armonk, NY, USA). Student's t test was used to compare two groups, and one-way ANOVA was used to compare multiple groups, $p$ value of less than 0.05 suggested statistical significance.

\section{RESULTS}

\section{ARF3 was downregulated in GC}

Firstly, the expression of ARF3 mRNA was determined in both human GC tissues and its corresponding normal tissues using qRT-PCR ( $\mathrm{n}=61$ for each group). We found that the expression of ARF3 mRNA was significantly downregulated in GC tissues than that in control group (Fig. 1A, $p<0.001$ ). Subsequently, expression of protein ARF3 was detected in GC tumor samples and normal samples using western blot ( $\mathrm{n}=4$ for each group) and IHC. As shown in Fig. $1 \mathrm{~B}$ and $1 \mathrm{C}$, it demonstrated that protein ARF3 was less expressed in GC tissues compared with normal tissue. Moreover, the expres- 
A

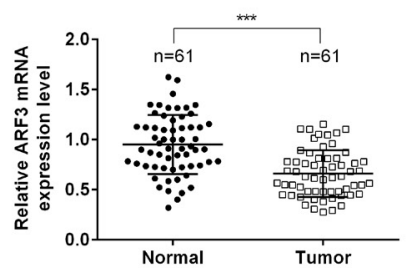

C

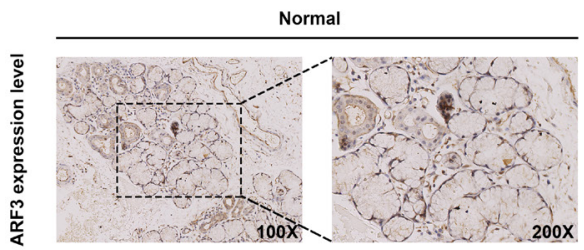

D

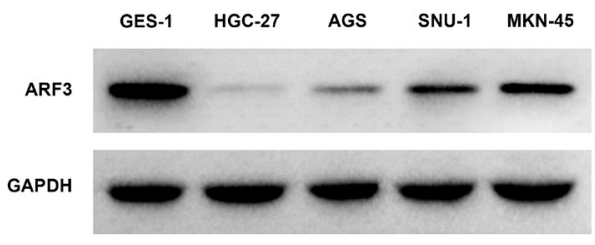

B
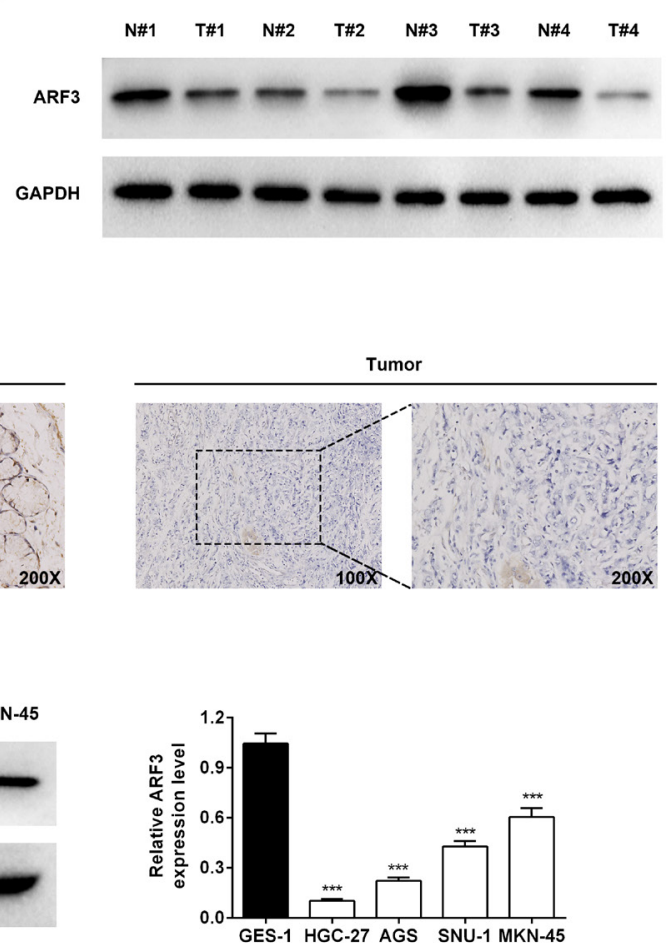

Figure 1. ARF3 was downregulated in GC tissues and cell lines.

(A) The expression of ARF3 mRNA was determined in human GC tumor tissues and control normal tissues ( $\mathrm{n}=61$ for each group) by qRTPCR. (B) The expression of ARF3 protein was tested in human GC tumor tissues and control normal tissues by western blot ( $n=4$ for each group). (C) The expression of ARF3 was detected human GC tumor tissues and control normal tissues by IHC. (D) The expression of ARF3 mRNA and protein was determined in GC cells (HGC-27, AGS, SNU-1, MKN-45) and normal human gastric mucosal cells (GES-1) by qRTPCR and western blot, respectively. Data were expressed as mean \pm S.D. ${ }^{* * *} p<0.001$ represent statistically difference.

sion of protein ARF3 was determined in GC cell lines (HGC-27, AGS, SNU-1, MKN-45) and normal human gastric mucosal cells (GES-1). Results demonstrated that ARF3 was remarkably less expressed in all GC cell lines. Among these cell lines, HGC-27 cells have the lowest level of ARF3, while MKN-45 cells expressed more ARF3 than other GC cells (Fig. 1D). In order to make the further results more convincing, we selected HGC27 and MKN-45 for subsequent experiments.
A

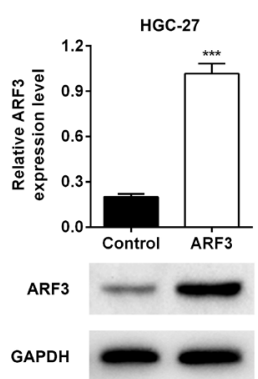

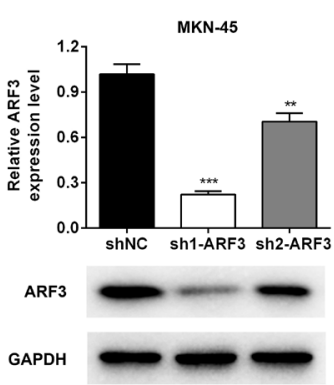

B

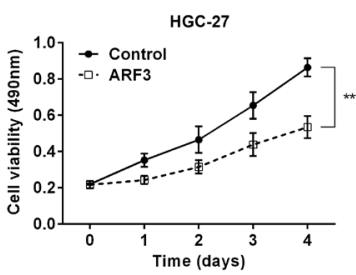

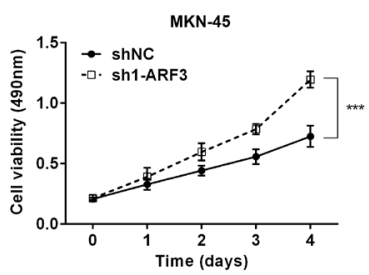

C
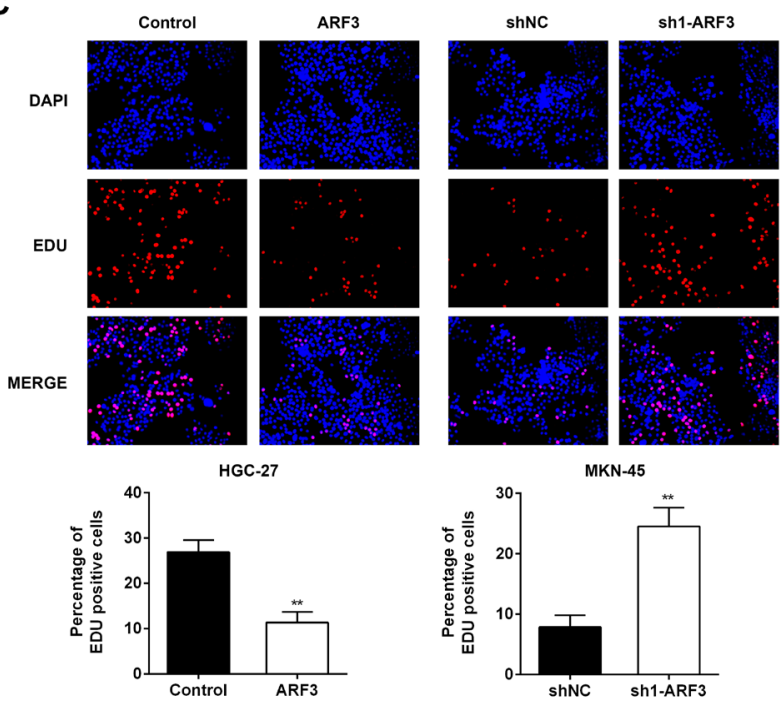

Figure 2. ARF3 suppressed the proliferation of GC cells.

(A) The transfect efficiency of pCDNA3.1-ARF3 plasmid and sh-ARF3 (sh1-ARF3, sh2-ARF3) were tested in HGC-27 and MKN-45 cells, respectively. (B) The cell viability in ARF3 overexpression and knockdown system was measured by CCK-8 assay. (C) Edu staining in both ARF3 overexpression and knockdown system. Data were expressed as mean \pm S.D. ${ }^{* *} p<0.01,{ }^{* * *} p<0.001$ represent statistically difference. 
A
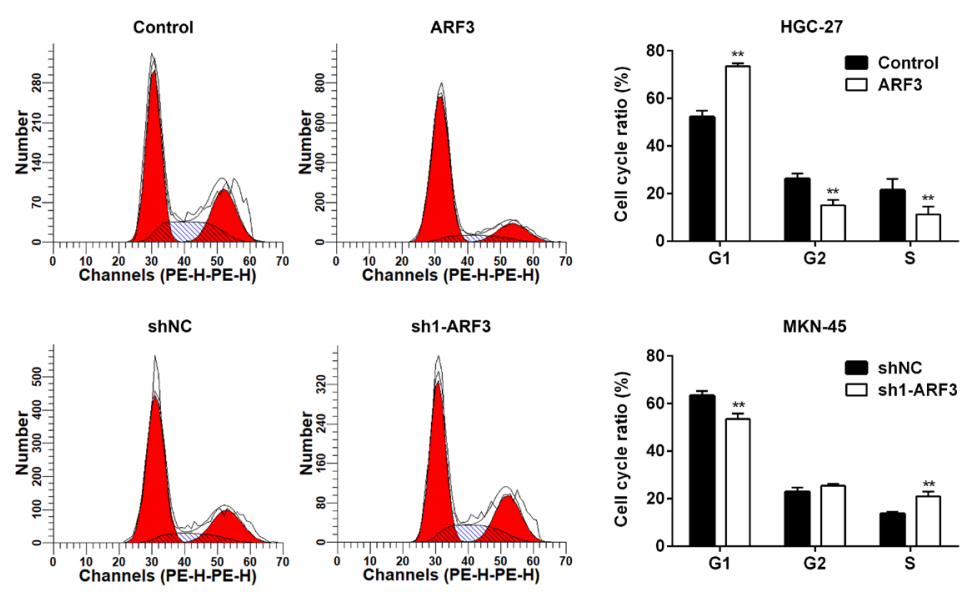

B
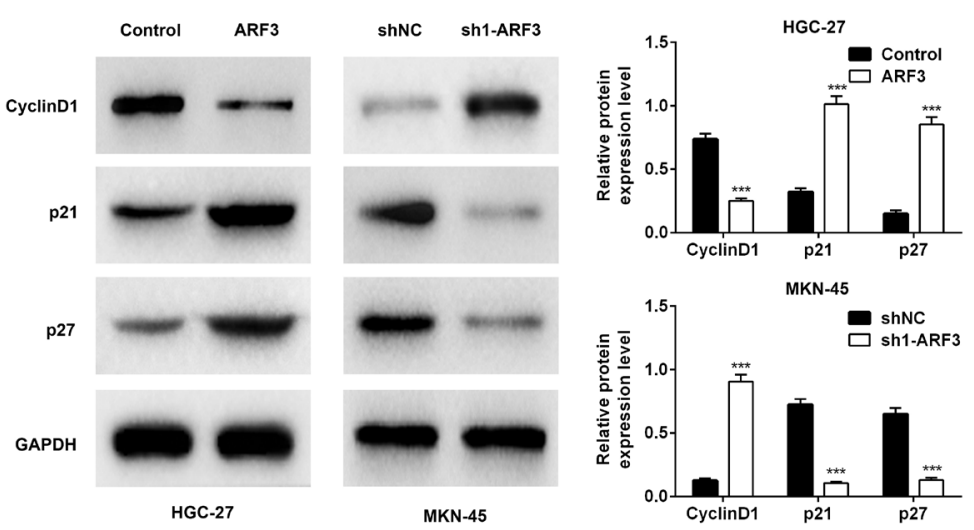

Figure 3. ARF3 induced cycle arrest of GC cells.

(A) The effect of ARF3 on GC cell cycle was determined in ARF3 overexpression and knockdown system by flow cytometer assay. (B) The expression of cyclinD1, p21 and p27 in ARF3 overexpression and knockdown system was determined by western blot. Data were expressed as mean \pm S.D. ${ }^{* *} p<0.01,{ }^{* * *} p<0.001$ represent statistically difference.

\section{ARF3 suppressed the proliferation of GC cells}

To identify the role of ARF3 in GC pathogenesis, pcDNA3.1-ARF3 plasmid and sh-ARF3 (sh1-ARF3, sh2-ARF3) have been used for ARF3 overexpression and knockdown in HGC-27 and MKN-45 cells, respectively. As shown in Fig. 2A, in HGC-27 cells, ARF3 $\mathrm{mRNA}$ and proteins were significantly upregulated after transfecting pcDNA3.1-ARF3 plasmid $(p<0.001)$, while in MKN-45 cells, expression of ARF3 was inhibited by sh1-ARF3 and sh2-ARF3 $(p<0.01, p<0.001)$. Due to the better inhibitory effect, sh1-ARF3 was used in further studies. Then, the effect of ARF3 on GC cell proliferation was determined by CCK-8 assay and Edu staining (Fig. 2B and 2C). Results demonstrated that overexpressed ARF3 could inhibit cell viability in a time dependent manner (Fig. 2B, $p<0.001$ ), while knockdown of ARF3 could promote cell viability (Fig. 2B, $p<0.001$ ). Furthermore, results of Edu staining showed that cells which overexpressed ARF3 have less Edu positive cells (Fig. 2C, $p<0.01$ ), while ARF3 knockdown cells have more Edu positive cells (Fig. 2C, $p<0.01$ ). These data demonstrated that ARF3 could suppress the proliferation of GC cells.

\section{ARF3 induced cycle arrest of GC cells}

Subsequently, the effect of ARF3 on GC cell cycle was determined in ARF3 overexpression and knockdown system, respectively. As shown in Fig. 3A, there was a significant amount of cell accumulation at G1 phase in ARF3 overexpressed group $(p<0.01)$, whereas on ARF3 knockdown group, cells accumulated less in the G1 phase while more gathered in $\mathrm{S}$ phase, suggesting that ARF3 has an effect on the GC cell cycle. Moreover, the expression of cell cycle related proteins including cyclinD1, p21 and p27 has been western blot tested. In Fig. 3B, we found that the expression of cyclinD1 was decreased in ARF3 overexpression cells $(p<0.001)$, while increased in ARF3 knockdown cells $(p<0.001)$. On the contrary, the expression of p21 and p27 was promoted in ARF3 overexpression cells $(p<0.001)$, while inhibited in ARF3 knockdown cells $(p<0.001)$. Taken together, these results indicated that ARF3 could induce cycle arrest of the GC cells.

\section{ARF3 promoted apoptosis of GC cells}

Further, GC cell apoptosis was detected in both ARF3 overexpression and knockdown system. As shown in Fig. 4A, in ARF3 overexpressed cells, apoptosis ratio was significantly increased compared to the control $(p<0.001)$, while knockdown of ARF3 had the opposite effect $(p<0.001)$. Furthermore, the levels of classic key factors of cell apoptosis (Bcl-2, Bax, Cleaved caspase-3) were investigated in each group. Results demonstrated that overexpressed ARF3 could inhibit the expression of $\mathrm{Bcl}-2(p<0.001)$, promote the expression of $\mathrm{Bax}$ and 
A
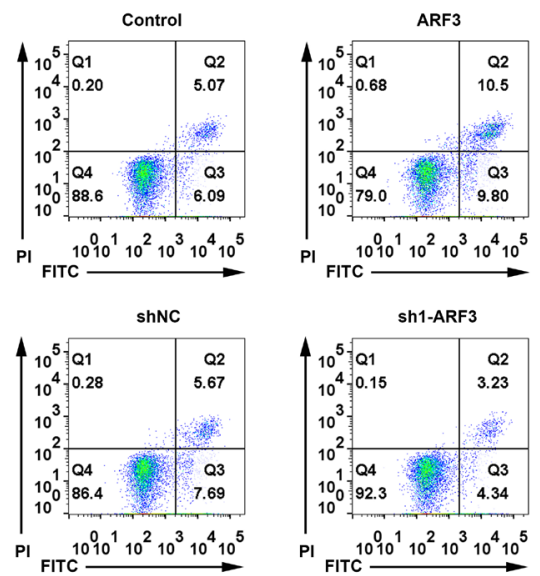

B
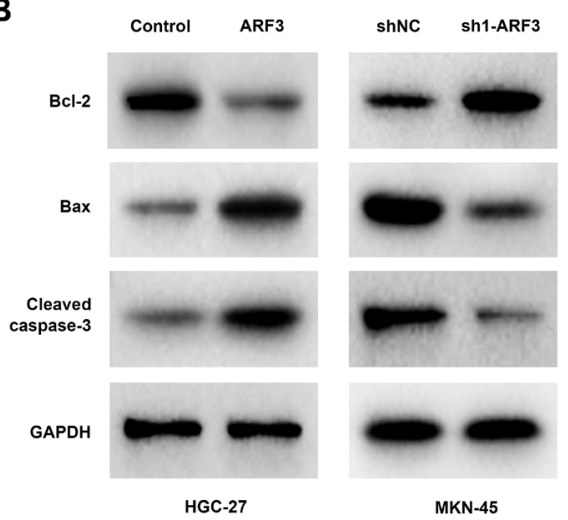
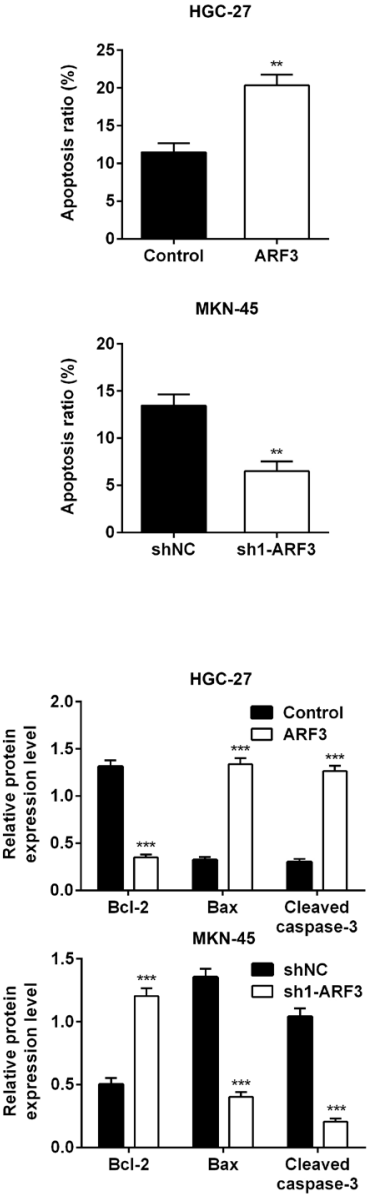

Figure 4. ARF3 promoted apoptosis of GC cells.

(A) The effect of ARF3 on GC cell apoptosis was determined in ARF3 overexpression and knockdown system by flow cytometer assay. Q1, necrotic cells; Q2, late apoptotic cells; Q3, early apoptotic cells; Q4, live cells (B) The expression of Bcl-2, Bax, Cleaved caspase-3 in ARF3 overexpression and knockdown system was determined by western blot. Data were expressed as mean \pm S.D. ${ }^{* *} p<0.01,{ }^{* * *} p<0.001$ represent statistically difference.

Cleaved caspase-3 $(p<0.001)$. However, knockdown of ARF3 had the opposite effect $(p<0.001)$.

\section{ARF3 regulated the activation of Akt and ERK pathway}

The expression of p-Akt, Akt, p-ERK1/2 and ERK1/2 were also tested in both ARF3 overexpression and knockdown system by western blot. We found that in ARF3 overexpressed cells, the expression of these factors was significantly decreased (Fig. 5, $p<0.001$ ). However, knockdown of ARF3 dramatically promoted the expression of these factors, suggesting that ARF3 could regulate the activation of Akt and ERK pathway (Fig. 5, $p<0.001)$.

\section{ARF3 inhibited tumorigenesis in vivo}

Finally, the effect of ARF3 on GC tumorigenesis was investigated by orthotopic xenograft mouse models in vivo. Male nude mice were injected with HGC-27 cells which transfected with pcDNA3.1-ARF3 plasmid or control vector. The pictures of tumor volume in each group were shown in Fig. 6A. For the quantification, we found that tumor volume in pcDNA3.1-ARF3 group were markedly smaller in each time point than that in the control group up to 18 days (Fig. 6B, $p<0.001$ ). The tumor weight in pcDNA3.1-ARF3 group were significantly lighter than that in the control (Fig. 6B, $p<0.001$, $\mathrm{n}=6)$. In IHC test, the expression of ARF3 was significantly increased in pcDNA3.1-ARF3 group, while the expression of $\mathrm{Ki}-67$ was decreased (Fig. 6C).

\section{DISCUSSION}

GC is the second leading cause of mortality with poor prognosis. Understanding the molecular mechanism of GC tumorigenesis is essential for the development of novel therapeutic options (Obermannová \& Lordick 2016). It has been previously reported that ARF3 acts as an important role in tumorigenesis in many types of cancers (Huang et al., 2019). In our study, we firstly found that ARF3 mRNA and protein was less expressed in human GC. Then, by using pcDNA3.1-ARF3 plasmid and sh-ARF3 (sh1-ARF3, sh2-ARF3), ARF3 overexpression and knockdown system was developed. Functional experiments showed that ARF3 suppressed the proliferation, induced cycle arrest and enhanced apoptosis of the GC cells. Our results demonstrated that in the GC development, ARF3 may play a tumor inhibitory factor.

Proliferation, cell cycle and apoptosis are vital features of cancer. Cell proliferation is an essential indicator for understanding the mechanisms of action of certain genes, proteins and pathways involved in cancer cell survival (Adan et al., 2016). For cell cycle progression, it can be divided into four phases: DNA synthesis (S), gap 1 

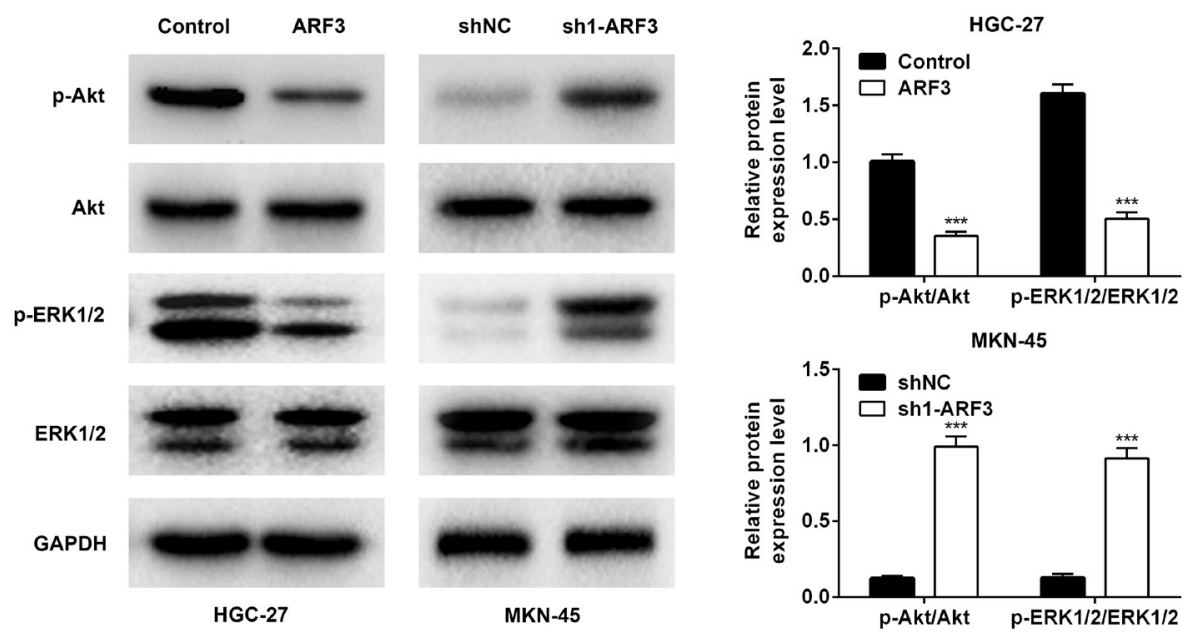

Figure 5. ARF3 regulated the activation of Akt and ERK pathway.

Western blot was conducted to determine the expression of p-Akt, Akt, p-ERK1/2 and ERK1/2 was in both ARF3 overexpression and knockdown system. Data were expressed as mean \pm S.D. ${ }^{* * *} p<0.001$ represent statistically difference.

A

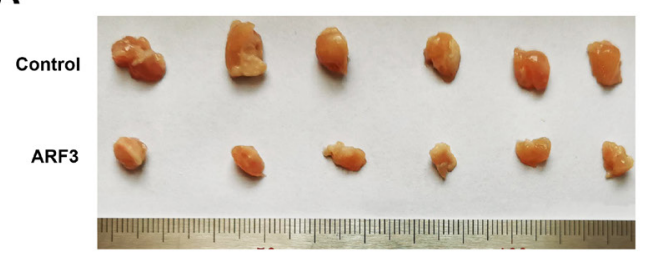

C

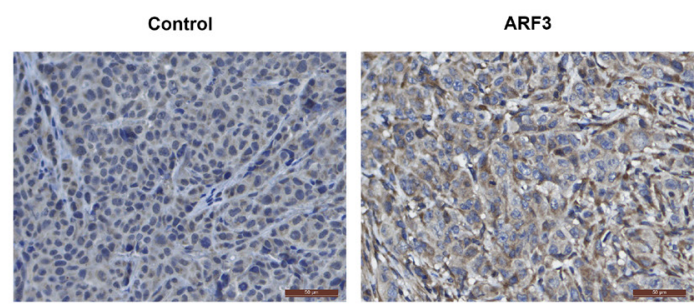

ARF3 expression level
B
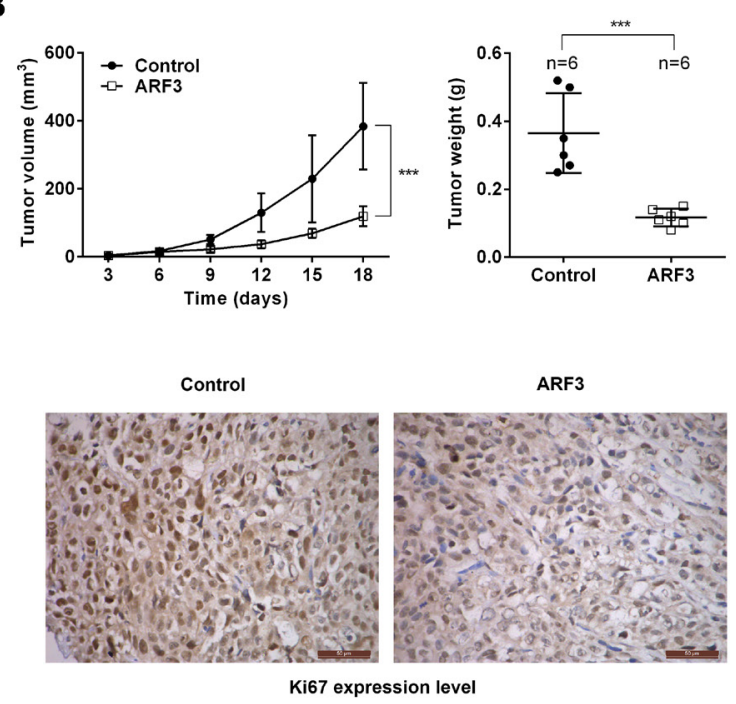

Figure 6. ARF3 inhibited tumorigenesis in vivo.

Mice treated with HGC-27 cell line transfected with pCDNA3.1-ARF3 plasmid or control vector. (A) Tumors collected from different groups of mice were shown. (B) The effect of ARF3 overexpression on tumor volume curve and tumor weight were analyzed. (C) The expression of ARF3 and Ki67 was detected by IHC assay in each group. Data were expressed as mean \pm S.D. ${ }^{* * *} p<0.001$ represent statistically difference.

(G1), gap 2 (G2) and mitosis (M) (Satyanarayana \& Kaldis 2009). Many factors were confirmed as a regulator in modulating cell cycle, for example D-type cyclins (D1, D2 and D3), cyclin-dependent kinase (CDKs), CIP/KIP family (p21, p27) and so on (Sherr 1994; Sherr 1995; Finn et al., 2016). As for apoptosis, it is considered to be a protective mechanism of the human body to purify cancer cells. Classically, Bcl-2 protein family (Bcl-2, Bax) regulate the release of cytochrome $\mathrm{c}$ from mitochondria, which is able to activate mitochondrial induced apoptosis pathway. In our study, it has been shown that ARF3 could suppress the proliferation of GC cells. Moreover, by performing flow cytometer assay and testing the expression of cyclinD1, p21 and p27, we found that ARF3 could arrest the cell cycle at G1 phase. For apoptosis, apoptotic cells in different groups were tested by flow cytometer assay. In parallel, the classical regulators in cell apoptosis including $\mathrm{Bcl}-2, \mathrm{Bax}$ and Cleaved Caspase-3 were detected in different groups. Results demonstrated that ARF3 could promote apoptosis of GC cells.

The AKT and ERK signaling pathways are important intracellular signal transduction cascades, regulating cell proliferation and growth, cell survival and apoptosis (Testa \& Bellacosa 2001; McCubrey et al., 2007; Manning \& Toker 2017). As a key node of PI3K/AKT signaling cascade, AKT, precisely excessive activated AKT, regulates the activity of each of the downstream factors, such as phosphorylate Ser2448 and m'TOR pathway (Sekulić et al., 2000; Mundi et al., 2016). For ERK signaling pathway, ERK1/2 is an important regulator and mediates various cellular processes such as activation of transcription factors, regulation of cell cycle and cell apoptosis (Murphy et al., 2002; Dhillon et al., 2007; Yang et al., 2013). The data of our study suggested that ARF3 could regulate the activation of AKT and ERK pathway, suggesting that the inhibitory role of ARF3 may exert by regulating AKT and 
ERK pathway. At the end of the experiment, we confirmed that ARF3 could inhibit tumorigenesis in vivo.

To sum up, our study provides the evidence of ARF3 being downregulated in the GC. Moreover, ARF3 acts as a regulator to suppress the proliferation, induce cycle arrest and promote apoptosis of GC cells. Mechanistically, ARF3 may inhibit proliferation and promotes apoptosis of GC by modulating AKT and ERK pathway. ARF3 might act as a potential biomarker for GC prognosis.

\section{Acknowledgements}

Not applicable.

\section{Funding}

Not applicable.

\section{Competing interests}

The authors state that there are no conflicts of interest to disclose.

\section{Ethics approval}

Ethical approval was obtained from the ethical committee of the Jiangsu Province Hospital of Chinese Medicine.

\section{Statement of Informed Consent}

Written informed consent was obtained from a legally authorized representative(s) for anonymized patient information to be published in this article.

\section{Availability of data and materials}

All data generated or analyzed during this study are included in this published article.

\section{Authors' contributions}

Jiayun Liu and Shenlin Liu designed the study, supervised the data collection, Xuequan Yao, Fukun Liu, Xiaoyu Wu and Guannan Wu analyzed the data, interpreted the data, Maolin Wu and Jing Deng prepare the manuscript for publication and reviewed the draft of the manuscript. All authors have read and approved the manuscript.

\section{REFERENCES}

Adan A, Kiraz Y, Baran Y (2016) Cell proliferation and cytotoxicity assays. Curr Pharm Biotechnol 17: 1213-1221. https://doi.org/10.2174/1 389201017666160808160513

Chang W, Ma L, Lin L, Gu L, Liu X, Cai H, Yu Y, Tan X, Zhai Y, Xu X, Zhang M, Wu L, Zhang H, Hou J, Wang H, Cao G (2009) Identification of novel hub genes associated with liver metastasis of gastric cancer. Int J Cancer 125: 2844-2853. https://doi. org/10.1002/ijc.24699

Charalampakis N, Economopoulou P, Kotsantis I, Tolia M, Schizas D, Liakakos T, Elimova E, Ajani JA, Psyrri A (2018) Medical management of gastric cancer: a 2017 update. Cancer Med 7: 123-133. https://doi.org/10.1002/cam4.1274

Dhillon AS, Hagan S, Rath O, Kolch W (2007) MAP kinase signalling pathways in cancer. Oncogene 26: 3279-3290. https://doi. org/10.1038/sj.onc.1210421

Ferro A, Peleteiro B, Malvezzi M, Bosetti C, Bertuccio P, Levi F, Negri E, La Vecchia C, Lunet N (2014) Worldwide trends in gastric cancer mortality (1980-2011), with predictions to 2015, and incidence by subtype. Eur J Cancer 50: 1330-1344. https://doi.org/10.1016/j. ejca.2014.01.029

Finn RS, Aleshin A, Slamon DJ (2016) Targeting the cyclin-dependent kinases (CDK) 4/6 in estrogen receptor-positive breast cancers. Breast Cancer Res 18: 17. https://doi.org/10.1186/s13058-015-0661-5

Fujiya K, Kawamura T, Omae K, Makuuchi R, Irino T, Tokunaga M, Tanizawa Y, Bando E, Terashima M (2018) Impact of malnutrition after gastrectomy for gastric cancer on long-term survival. Ann Surg Oncol 25: 974-983. https://doi.org/10.1245/s10434-018-6342-8

Huang D, Pei Y, Dai C, Huang Y, Chen H, Chen X, Zhang X, Lin C, Wang H, Zhang R, Wan X, Wang L (2019) Up-regulated ADP-Ribosylation factor 3 promotes breast cancer cell proliferation through the participation of FOXO1. Exp Cell Res 384: 111624. https://doi. org/10.1016/j.yexcr.2019.111624

Kahn RA, Cherfils J, Elias M, Lovering RC, Munro S, Schurmann A (2006) Nomenclature for the human Arf family of GTP-binding proteins: ARF, ARL, and SAR proteins. J Cell Biol 172: 645-650. https://doi.org/10.1083/jcb.200512057

Kondo Y, Hanai A, Nakai W, Katoh Y, Nakayama K, Shin HW (2012) ARF1 and ARF3 are required for the integrity of recycling endosomes and the recycling pathway. Cell Struct Funct 37: 141-154. https://doi.org/10.1247/csf.12015

Li F, Mandal M, Mishra SK, Barnes CJ, Kumar R (2002) Heregulin promotes expression and subcellular redistribution of ADP-ribosylation factor 3. FEBS Lett 524: 49-53. https://doi.org/10.1016/ s0014-5793(02)02994-0

Lunet N, Lacerda-Vieira A, Barros H (2005) Fruit and vegetables consumption and gastric cancer: a systematic review and meta-analysis of cohort studies. Nutr Cancer 53: 1-10. https://doi.org/10.1207/ s15327914nc5301_1

Manning BD, Toker A (2017) AKT/PKB Signaling: navigating the network. Cell 169: 381-405. https://doi.org/10.1016/j.cell.2017.04.001

McCubrey JA, Steelman LS, Chappell WH, Abrams SL, Wong EW, Chang F, Lehmann B, Terrian DM, Milella M, Tafuri A, Stivala F, Libra M, Basecke J, Evangelisti C, Martelli AM, Franklin RA (2007) Roles of the Raf/MEK/ERK pathway in cell growth, malignant transformation and drug resistance. Biochim Biophys Acta 1773: 12631284. https://doi.org/10.1016/j.bbamcr.2006.10.001

Mundi PS, Sachdev J, McCourt C, Kalinsky K (2016) AKT in cancer: new molecular insights and advances in drug development. Br J Clin Pharmacol 82: 943-956. https://doi.org/10.1111/bcp.13021

Murphy LO, Smith S, Chen RH, Fingar DC, Blenis J (2002) Molecular interpretation of ERK signal duration by immediate early gene products. Nat Cell Biol 4: 556-564. https://doi.org/10.1038/ncb822

Obermannová R, Lordick F (2016) Insights into next developments in advanced gastric cancer. Curr Opin Oncol 28: 367-375. https://doi. org/10.1097/cco.0000000000000289

Peleteiro B, La Vecchia C, Lunet N (2012) The role of Helicobacter pylori infection in the web of gastric cancer causation. Eur I Cancer Prev 21: 118-125. https://doi.org/10.1097/CEJ.0b013e32834a7f66

Satyanarayana A, Kaldis P (2009) Mammalian cell-cycle regulation: several Cdks, numerous cyclins and diverse compensatory mechanisms. Oncogene 28: 2925-2939. https://doi.org/10.1038/onc.2009.170

Sekulić A, Hudson CC, Homme JL, Yin P, Otterness DM, Karnitz LM, Abraham RT (2000) A direct linkage between the phosphoinositide 3-kinase-AKT signaling pathway and the mammalian target of rapamycin in mitogen-stimulated and transformed cells. Cancer Res 60: 3504-3513.

Seo J, Seong D, Lee SR, Oh DB, Song J (2020) Post-translational regulation of ARF: perspective in cancer. Biomolecules 10: https://doi. org/10.3390/biom10081143

Sherr CJ (1995) D-type cyclins. Trends Biochem Sci 20: 187-190. https:// doi.org/10.1016/s0968-0004(00)89005-2

Sherr CJ (1994) Growth factor-regulated G1 cyclins. Stem Cells 12 (Suppl 1): 47-55; discussion 55-47.

Smith SA, Holik PR, Stevens J, Melis R, White R, Albertsen H (1995) Isolation and mapping of a gene encoding a novel human ADP-ribosylation factor on chromosome 17q12-q21. Genomics 28: 113-115. https://doi.org/10.1006/geno.1995.1115

Strong VE (2018) Progress in gastric cancer. Updates in Surgery 70: 157159. https://doi.org/10.1007/s13304-018-0543-3

ztul E, Chen PW, Casanova JE, Cherfils J, Dacks JB, Lambright DG, Lee FS, Randazzo PA, Santy LC, Schürmann A, Wilhelmi I, Yohe ME, Kahn RA (2019) ARF GTPases and their GEFs and GAPs: concepts and challenges. Mol Biol Cell 30: 1249-1271. https://doi. org/10.1091/mbc.E18-12-0820

Testa JR, Bellacosa A (2001) AKT plays a central role in tumorigenesis. Proc Natl Acad Sci US A 98: 10983-10985. https://doi. org/10.1073/pnas.211430998

Yang SH, Sharrocks AD, Whitmarsh AJ (2013) MAP kinase signalling cascades and transcriptional regulation. Gene 513: 1-13. https://doi. org/10.1016/j.gene.2012.10.033

Zhang J, Zhou YJ, Yu ZH, Chen AX, Yu Y, Wang X, Cao XC (2019) Identification of core genes and clinical roles in pregnancy-associated breast cancer based on integrated analysis of different microarray profile datasets. Biosci Rep 39: https://doi.org/10.1042/bsr20190019

Zhang Z, H Li, Y Zhao, Q Guo, Y Yu, S Zhu, S Zhang, L Min,P $\mathrm{Li}$ (2019) Asporin promotes cell proliferation via interacting with PSMD2 in gastric cancer. Frontiers Biosci (Landmark edn). 24: 11781189. PMID: 31136974 